\title{
Assessment and management of congenital heart disease in the newborn by the district paediatrician
}

\author{
Eric D Silove
}

In the newborn, congenital heart disease is often life threatening and may have a dramatic presentation. Most textbooks have a systematic approach to congenital heart disease in general and fail to create an awareness of the major differences of presentation that occur in the newborn compared with the older infant. It is my purpose to alert paediatricians to their critical role in reducing the mortality and morbidity which continue to result from late recognition, referral, and management. This article is inspired by my observations over many years of both triumphs and disasters in the success and failure of early recognition and management of such newborns. It is intended to be provocative and my approach will be unorthodox because I believe that conventional teaching has not always succeeded in ensuring that available resources are used optimally.

In order to help with a better understanding of the problems, I will first highlight some physiological mechanisms during the transition from fetal to neonatal life and will then lead on to a recognition of early physical signs. Then the common groups of lesions will be described, and the importance of their early management will be discussed. It will be stressed throughout that the district paediatrician should have a high index of suspicion and a low threshold for immediate intervention and referral of any newborn in whom congenital heart disease is considered.

\section{Changes in the early neonatal period ${ }^{1}$}

In the normal fetal heart blood flows from the left ventricle directly into the aorta through the aortic valve, and from the right ventricle through the pulmonary valve predominantly into the descending aorta via the arterial duct, only about $10 \%$ of the output passing to the lungs. The pressures in the two ventricles are identical. The first breath expands not only the air capacity of the lungs but also the pulmonary vascular bed. The small pulmonary arteries (the resistance vessels) have a muscular media similar to that found in systemic arteries and they are constricted during fetal life. The sudden increase in alveolar oxygen tension relaxes these vessels and over several months they 'mature' into the thinner walled 'adult' pulmonary arteries. The arterial duct, through which $90 \%$ of right ventricular blood flowed during fetal life, constricts in response to the raised alveolar oxygen tension. At this early stage flow through the arterial duct already tends to pass from aorta to pulmonary artery. The fall in pulmonary vascular resistance and the increased return of blood from the lungs to the left atrium, tends to reduce flow from the right atrium to left atrium through the foramen ovale which undergoes functional closure. Consequently, many of the congenital abnormalities that the fetus survived become life threatening in the newborn period because one or more of these events has occurred. The timing is unpredictable, which accounts for the presentation of some neonates with a given lesion during the first few days of life, and others not until a few weeks later.

Obstructive lesions of the right heart result in a reduced pulmonary blood flow which may become critical when the arterial duct closes, especially when it is the only or main source of blood supply to the pulmonary arteries. Similarly, left heart obstruction may severely reduce blood flow to the kidneys and other organs, the perfusion of which might be dependent on flow from the pulmonary artery through a patent arterial duct to the aorta. In a variety of anomalies such as transposition of the great arteries, tricuspid atresia or mitral atresia, closure of the foramen ovale may prevent the life dependent flow of blood through it in either direction. The early fall in pulmonary vascular resistance might encourage a high pulmonary blood flow in certain lesions with a 'left-to-right shunt' and cause early volume overload of the systemic ventricle.

\section{Recognition of early physical signs in congenital heart disease}

There are some early signs which should alert the paediatrician to the possibility of congenital heart disease. The most dramatic is the presence of cyanosis which immediately leads one to consider the differentiation from respiratory causes, from persistent fetal circulation and from other less common problems. Blue newborns with congenital heart disease are usually not breathless unless there is accompanying acidosis or pulmonary oedema. In contrast, those with primary respiratory 
problems are invariably tachypnoeic. It may be very difficult to distinguish the neonate with perinatal asphyxia from one with congenital heart disease because the former may have heart failure and cyanosis with evidence of cardiomegaly and the murmur of tricuspid incompetence.

Cyanosis is readily confirmed by pulse oximetry, and the administration of $100 \%$ oxygen for 8-10 minutes will usually increase the arterial oxygen saturation $\left(\mathrm{SaO}_{2}\right)$ to at least $95 \%$ if the aetiology is respiratory and occasionally if there is persistent fetal circulation. If the $\mathrm{SaO}_{2}$ does increase in this way, it is important to measure the arterial oxygen tension $\left(\mathrm{PaO}_{2}\right)$, preferably from a blood sample but reasonably reliably by transcutaneous electrode. $\mathrm{A} \mathrm{PaO}_{2}$ as low as $6 \mathrm{kPa}$ can result in the $\mathrm{SaO}_{2}$ reaching $95 \%$ because fetal haemoglobin results in a marked shift of the oxygen dissociation curve to the left. ${ }^{2}$ In general, the hyperoxia test should achieve a $\mathrm{PaO}_{2}$ of at least $20 \mathrm{kPa}$ in order to exclude a significant right to left shunt, and presumably cyanotic congenital heart disease. Arterial blood measurements should preferably be made from the right upper limb and transcutaneous measurements from the right upper thoracic quadrant in order to reduce the possibility of sampling deoxygenated pulmonary arterial blood that has passed through the arterial duct into the descending aorta. A raised arterial carbon dioxide tension $\left(\mathrm{PaCO}_{2}\right)$ is usually not found in congenital heart disease. Deep cyanosis is usually very obvious but mild cyanosis, reducing the $\mathrm{SaO}_{2}$ to around $80 \%$ is much more subtle. At the slightest suspicion, pulse oximetry measurements should be made. In the absence of clear cut evidence of a primary respiratory cause of the cyanosis, advice should be sought from an appropriate paediatric cardiology referral centre before transfer.

An important but often neglected early physical sign of serious congenital heart disease is difficulty in feeding. It is usually the first sign of cardiac failure, invariably accompanied by tachypnoea. The old adage, 'listen to the patient', is seldom more important than when a mother complains that her baby is not taking its feeds or is feeding very slowly, possibly breathing fast and often sweating. If a baby takes longer than 30 minutes for a feed, dyspnoea should be suspected. Its importance should be impressed upon nurses, health visitors, and junior medical staff. Careful observation of the breathing pattern at this early stage will often reveal slight subcostal recession. The liver might not yet be enlarged. A chest radiograph will invariably reveal cardiac enlargement and it is essential that a good inspiratory film is obtained if this sign is to be demonstrated reliably. Mild cyanosis may accompany the respiratory difficulty associated with cardiac failure even in those conditions which are conventionally classified as 'acyanotic'. In a breathless, blue neonate thought to have a primary lung condition, obstructed total anomalous pulmonary venous return should always be considered. The dyspnoea is due to pulmonary oedema but there is no cardiac enlargement on chest radiograph. The radiographic sign of pulmonary oedema is characteristic but may be difficult to distinguish from hyaline membrane disease.

A cardiac murmur should always be notified to a paediatric cardiology referral centre at an early stage. Its intensity and length may have no bearing on the severity of the lesion. For example, many newborns with large ventricular septal defect may have a barely audible murmur. A discussion with the paediatric cardiologist will usually be followed by an early visit for echocardiography. It is far preferable to err on the side of caution and refer early some relatively innocent murmurs, than to risk the consequences of failing to refer a critical lesion.

It is fundamental to palpate the pulses in all newborns. Positive identification of the femoral and the brachial pulses must be made. In some critical left heart obstructive lesions the femoral pulses might be palpable but one or both of the brachials may be absent. It is also important to recognise that all of the pulses might be palpable a few days after birth, even in the presence of severe left heart obstructive lesions and that some or all may then disappear when the arterial duct closes, usually heralded by feeding difficulty and other evidence of pulmonary oedema.

In some neonates circulatory collapse may be the presenting feature either because some of the early signs have not been recognised or because they were not present at the time the baby was discharged from hospital. There is invariably a preceding history of feeding difficulty, often for just 6-12 hours. The baby has weak or absent pulses, is tachypnoeic with recession and usually has an enlarged liver. Arterial blood gases usually reveal significant acidosis, the base deficit being at least 8 $\mathrm{mmol} / \mathrm{l}$. The $\mathrm{PaCO}_{2}$ may not be raised and the $\mathrm{PaO}_{2}$ only slightly reduced. All too often, these infants are initially investigated and treated for septicaemia before the possibility of congenital heart disease is considered. Cardiac enlargement on a good chest radiograph should lead the paediatrician to seek urgent advice from a paediatric cardiology centre.

It is debatable whether the time should be taken to do an electrocardiogram (ECG) before obtaining cardiological advice except when an arrhythmia is suspected. Supraventricular tachycardia or heart block may present either in utero or soon after birth. An ECG tracing is then essential in the neonate and if its interpretation is difficult, it can be faxed to the cardiology centre for advice on early management before transfer. In structural heart anomalies a technically adequate ECG and the correct interpretation of the electrical axis and of the presence of left or right ventricular hypertrophy might give some clue to the type of lesion. However, a normal tracing should never preclude early referral. Similarly, echocardiography in the peripheral neonatal unit can waste precious time and be very misleading unless it has been performed by someone highly skilled and trained in congenital heart ultrasound. 


\section{Comments about specific anomalies}

It is not my intention to provide a comprehensive account of each of the congenital heart anomalies but, instead, to focus attention on some specific points which might be helpful to the district paediatrician.

\section{CYANOTIC ANOMALIES}

Once the presence of cyanosis has been confirmed and been considered to be cardiac in origin, some generalisations may be made about groups of abnormalities. Obstructive right heart lesions might or might not be associated with a cardiac murmur, the genesis of which might be difficult to determine. For example, it might arise from pulmonary or subpulmonary stenosis or from tricuspid regurgitation, or from a patent ductus arteriosus. On clinical grounds it is difficult to be certain and it is therefore unimportant. A chest radiograph is more helpful because it will usually show a normal size heart and decreased lung vascularity. There are descriptions of the cardiac contour which are said to be 'classical' but seem to apply in very few cases. The ECG may be normal in Fallot's tetralogy type lesions. There may be a leftward axis and left ventricular hypertrophy when there is hypoplasia of the right ventricle such as in tricuspid atresia or in pulmonary atresia with intact ventricular septum.

In cyanotic anomalies with an increased pulmonary blood flow the chest radiograph usually shows cardiac enlargement and there may be increased pulmonary vascularity. Examples include transposition of the great arteries though the 'typical egg-on-side' may not be apparent, and other less common lesions such as univentricular atrioventricular connection, persistent truncus arteriosus (in which the cyanosis may be extremely mild), and total anomalous pulmonary venous return in which cyanosis might not be detectable unless the pulmonary veins are obstructed. Although hypoplastic left heart syndrome is an obstructive left heart lesion, there is an obligatory right to left shunt and the infants are therefore cyanosed and also have an increased pulmonary blood flow.

\section{ACYANOTIC ANOMALIES}

For convenience these are usually divided into the obstructive left heart lesions and those with large left to right shunts but there is considerable overlap with the 'cyanotic anomalies'. The predominant clinical feature in the significant 'acyanotic anomalies' is the presence of cardiac failure which has been described above. The importance of palpating the femoral and brachial pulses cannot be over emphasised. For example, coarctation of the aorta is classically characterised by good brachial pulses and absent or weak femorals. Some forms of interruption of the aortic arch may be associated with a good right brachial pulse but absent left brachial and weak femorals. The hypoplastic left heart syndrome is usually associated with weak or absent brachial and femoral pulses and sometimes the femorals are more easily palpable than the brachials because the predominant flow into the aorta through the arterial duct passes into the descending aorta. Similarly, critical aortic stenosis may be associated with reasonable femoral pulses but absent brachials due to the same mechanism. It is common for no murmur to be audible in any of the obstructive left heart lesions, including critical aortic stenosis and in general a murmur should be regarded as a 'soft' physical sign. The chest radiograph will usually show considerable cardiac enlargement, usually due to dilatation of the right heart chambers and there will be evidence of pulmonary oedema. On the ECG there is usually right ventricular hypertrophy in aortic arch lesions due to left to right atrial shunting and pulmonary hypertensive right heart failure. ${ }^{3}$ In some cases of critical aortic stenosis it shows left ventricular strain.

The common left to right shunts causing significant problems in the neonate include large ventricular septal defect, patent arterial duct, complete atrioventricular septal defect, and persistent truncus arteriosus. The latter is often classified as a 'cyanotic' lesion but cyanosis is usually very mild and the very high pulmonary blood flow predominates. In all these lesions, the chest radiograph shows cardiac enlargement and increased lung vascularity. The ECG may show left ventricular dominance and in the case of atrioventricular septal defect the axis is 'superior'. Mild cyanosis may be present due to pulmonary oedema but the $\mathrm{SaO}_{2}$ will usually rise to normal values during the administration of oxygen. Although persistent arterial duct usually presents in the preterm infant, it should be considered in the differential diagnosis of any infant with cardiac failure and will often be found in combination with other anomalies. The diagnosis of persistent arterial duct in the preterm infant is usually made quite readily on a neonatal unit and it falls into a different category from the types of anomaly which are the subject of this article.

Tachyarrhythmias often cause severe cardiac failure in the neonate and should be diagnosed readily. Cardiomyopathies causing cardiac failure might be mistaken for one of the obstructive left heart lesions because the neonates often have very weak pulses, severe cardiac failure, and on chest radiograph have severe cardiac enlargement and pulmonary oedema without an increase in lung vascularity.

\section{Early management of congenital heart disease}

Most infants with cyanotic congenital heart anomalies will benefit if patency of the arterial duct is maintained. This principle applies in all right heart obstructive lesions such as pulmonary atresia, tricuspid atresia, and severe Fallot's tetralogy. Ductal patency is also of value in transposition of the great arteries because the increase in pulmonary blood flow results in an increased volume of blood returning from the left atrium, thereby raising the left 
atrial pressure and encouraging shunting of oxygenated blood through the foramen ovale into the right heart and thence to the aorta. Newborns with obstructive left heart lesions such as coarctation of the aorta, interrupted aortic arch, critical aortic stenosis, and hypoplastic left heart syndrome also benefit from maintained ductal patency. It promotes flow into the descending aorta and especially to the kidneys, thereby delaying or preventing the development of a metabolic acidosis.

The early use of prostaglandins $E_{1}$ or $E_{2}$ $\left(\mathrm{PGE}_{1}\right.$ or $\left.\mathrm{PGE}_{2}\right)$ may therefore be advisable before transfer to the cardiology centre. It is important first to discuss this question with the paediatric cardiologist and to confirm dosage. Usually it is safe to start intravenous $\mathrm{PGE}_{1}$ or $\mathrm{PGE}_{2}$ in a dose of $0.005 \mu \mathrm{g} / \mathrm{kg} / \mathrm{min}{ }^{4}{ }^{4} \mathrm{recom}-$ mend diluting $500 \mu \mathrm{g}$ in $500 \mathrm{ml}$ of $5 \%$ dextrose, giving a concentration of $1 \mu \mathrm{g} / \mathrm{ml}$. The infusion rate will then be $0.3 \mathrm{ml} / \mathrm{kg} /$ hour (or $0.9 \mathrm{ml} /$ hour for a $3000 \mathrm{~g}$ infant). If the $\mathrm{SaO}_{2}$ has not increased within 20 minutes, the dose may be doubled and will still be only 10-20\% of the dose recommended by the manufacturers. Even at this low dosage, apnoea can be a complication and it may therefore be advisable in some cases to perform endotracheal intubation and ventilation before transfer, again having first obtained the agreement of the cardiac centre.

Most infants with cardiac failure, and especially those with an obstructive left heart lesion, require supportive treatment before and during transfer to a cardiac centre. They will often deteriorate significantly while waiting for an ambulance and during transfer. They should be treated in the same way as any other neonate who has shown evidence of circulatory collapse, for example, those with fulminating sepsis. Blood gas and acid-base measurements should be obtained and repeated on several occasions. If the base deficit is less than 8 $\mathrm{mmol} / \mathrm{l}$, the measurements should be repeated within half an hour and if the base deficit has increased, it should be 'half corrected'. At the same time, endotracheal intubation and ventilatory support should be undertaken if the acidosis is increasing, even if the $\mathrm{PaCO}_{2}$ is normal. A neonate who develops metabolic acidosis as a result of cardiac failure needs urgent ventilatory support, partly to prevent worsening of the acidosis and partly to treat the pulmonary oedema. The acidosis increases largely due to the inadequate perfusion of the kidneys but also as a result of the infant becoming exhausted in its respiratory effort.

An exhausted, acidotic neonate will have had a decreased fluid intake for many hours and will invariably be hypovolaemic even though it is in cardiac failure. When ventilation has been controlled and acidosis partly corrected, the infusion of plasma or human albu$\mathrm{min}(5 \%$ in saline) in a dose of $5-10 \mathrm{ml} / \mathrm{kg}$ should be undertaken. At the same time, dobutamine infusion should be commenced in a dose of $7-10 \mu \mathrm{g} / \mathrm{kg} / \mathrm{min}$ via a peripheral intravenous cannula. If it is possible to introduce a central venous line, it is helpful to add dopamine in a dose of $3 \mu \mathrm{g} / \mathrm{kg} / \mathrm{min}$ in order to improve renal perfusion. When the peripheral pulses have improved and skin temperature increased, frusemide $1 \mathrm{mg} / \mathrm{kg}$ may be given intravenously to reduce the increased extravascular fluid but repeated doses of frusemide are not advisable until renal perfusion is adequate. No neonate in circulatory collapse or in a severely acidotic state should be transferred to a cardiac centre until supportive measures have been instituted and the infant has shown some signs of stabilising. The management will usually be undertaken in close consultation with the paediatric cardiologist and some of the major centres may have an intensive care team that is geared to go out to the peripheral hospital in order to help stabilise the neonate and to supervise its transportation.

Supraventricular tachycardia might beneficially be treated by the district paediatrician and the same supportive measures should be taken if the infant is in severe cardiac failure. After discussing the management with a paediatric cardiologist, an initial dose of intravenous digoxin $5 \mu \mathrm{g} / \mathrm{kg}$ may be given followed 10-15 minutes later by a rapid bolus of adenosine using the appropriate dosage regimen. ${ }^{5}$ While adenosine alone might convert the supraventricular tachycardia into sinus rhythm, reversion into supraventricular tachycardia often follows within seconds or minutes and another drug will usually be necessary to ensure that sinus rhythm is maintained. Early transfer of unresponsive cases should be arranged with the referral centre.

It is hoped that an increased awareness of congenital heart disease and an aggressive approach to its early management will help to reduce the significant mortality and morbidity. The health care professionals who are closest to the problem are not only the district paediatricians but, equally important, the junior medical staff, general practitioners, midwives, nurses, and health visitors. It is important that they should be informed of the early presenting signs of congenital heart disease in the newborn.

1 Rudolph AM. The changes in the circulation after birth Their importance in congenital heart disease. Circulation 1970; 41: 343-59.

2 Delivoria-Papadopoulos $M$, Roncevic NP, Oski FA. Postnatal changes in oxygen transport of term, premature, and sick infants: the role of red cell 2,3 - diphosphoglycerate and adult hemoglobin. Pediatr Res 1971; 5: 235-45. 3 Graham TP Jr, Atwood GF, Boerth RC, Boucek RJ Jr, Smith CW. Right and left heart size and function in infants with symptomatic coarctation. Circulation 1977; 56: $641-7$.

4 Silove ED, Roberts DGV, De Giovanni JV. Evaluation of oral and low dose intravenous prostaglandin $\mathrm{E}_{2}$ in management of ductus dependent congenital heart disease. Arch Dis Child 1985; 60: 1025-30.

5 Clarke B, Till JA, Rowland E, Ward DE, Barnes PJ, Shinebourne EA. Rapid and safe termination of supraventricular tachycardia in children by adenosine. Lancet 1987 ; i: $229-301$.

\section{Further reading}

Haworth SG, Bull C. Physiology of congenital heart disease. Arch Dis Child 1993; 68: 707-11. 